Eur. J. Clin. Chem. Clin. Biochem.

Vol. 32, 1994, pp. 473-478

(C) 1994 Walter de Gruyter \& Co.

Berlin · New York

\title{
Pitfalls in the Differentiation of N-Glycosylation Variants of Prostate-Specific Antigen Using Concanavalin A
}

\author{
By Marja P. van Dieijen-Visser ${ }^{1}, J$. van Pelt ${ }^{1}$ and K. P. J. Delaere ${ }^{2}$
}

1 Department of Clinical Chemistry

2 Department of Urology

De Wever Hospital, Heerlen, The Netherlands

(Received October 22, 1993/February 21, 1994)

Summary: We determined the optimal conditions for the separation of $\mathrm{N}$-glycosylation variants of prostate-specific antigen using concanavalin $\mathrm{A}$. Concanavalin $\mathrm{A}$ is a lectin that binds to the terminal sugar residues of glycoproteins. We demonstrated that differences in the percentage of prostate-specific antigen bound to concanavalin A-Sepharose in patients with benign prostatic hyperplasia compared with patients with prostatic carcinoma, as described in the literature, arise when insufficient concanavalin A binding sites are added for complete binding of the glycosylation variants of prostate-specific antigen.

We observed similar percentages of prostate-specific antigen bound to concanavalin A-Sepharose for benign prostatic hyperplasia $(86.3 \% \pm 7.5$, mean \pm SD) and carcinoma patients $(81.8 \% \pm 12.0$, mean \pm SD), when sufficient concanavalin A-Sepharose was added to allow optimal binding, and when samples with high prostate-specific antigen concentrations were not pre-diluted before incubation with concanavalin A-Sepharose.

We conclude that differentiation of patients with benign prostatic hyperplasia or carcinoma of the prostate on the basis of differences in percentages of prostate-specific antigen bound to concanavalin A-Sepharose, i. e. separation of $\mathrm{N}$-glycosylation variants, is not possible.

\section{Introduction}

Prostate-specific antigen is a glycoprotein produced in the cytoplasm of both benign and malignant prostate cells. Its unique organ specificity established prostatespecific antigen as the most sensitive marker of prostatic cancer. However, benign prostatic hyperplasia, a common condition in elderly, is also associated with a slight elevation of the prostate-specific antigen concentration $(1,2)$.

Barak et al. (3) observed different N-glycosylation variants of prostate-specific antigen in patients with benign prostatic hyperplasia compared with patients with carcinoma of the prostate. The authors presented a complete differentiation of benign prostatic hyperplasia and prostate cancer patients on the basis of percentages of prostate-specific antigen bound to concanavalin A-Se- pharose. Chan et al. (4) observed a significant difference $(p<0.001)$, but with a considerable overlap, in the distribution of the concanavalin A non-reactive fraction of prostate-specific antigen for benign prostatic hyperplasia and prostate cancer patients. Different procedures were used in these studies. Marrink et al. (5) recently reported that there is no striking difference in the glycosylation pattern of prostate-specific antigen in benign or malignant prostate disease and that any binding ratio can be found. We indicated that the conclusions might be influenced by the experimental conditions used, like insufficient correction for dilution effects (6).

Changes in the $\mathrm{N}$-glycosylation of proteins in cancer and their investigation using lectins have recently been extensively reviewed (7). It is clear that differences in glycosylation could provide the basis for more sensitive and 
more discriminative clinical tests. However, it should be realised that the non-covalent lectin-carbohydrate interactions will be influenced by various experimental variables and therefore the search for optimal assay conditions is of utmost importance.

Here, we determined the optimal conditions for separation of the molecular variants of prostate-specific antigen using concanavalin A-Sepharose. We examined discrepancies in the literature concerning differences in the $\mathrm{N}$-glycosylation of prostate-specific antigen variants in patients with benign prostatic hyperplasia and patients with cancer of the prostate.

\section{Materials and Methods}

\section{Subjects and specimens}

Blood specimens were collected from males attending the Urology Department of De Wever Hospital in Heerlen (NL) with the presumptive diagnosis of carcinoma of the prostate. Specimens were collected before rectal examination. The diagnosis of prostate cancer was established histologically, whereas the diagnosis of benign prostatic hyperplasia was made on a clinical basis (history, rectal palpation, endoscopy). Bone metastases were established by bone scan. The cancer group was classified according to the TNM system of the Union International Contre le Cancer. Sera of 16 prostatic cancer patients (untreated) and 77 patients with benign prostatic hypertrophy were frozen at $-20^{\circ} \mathrm{C}$. Patients with prostatespecific antigen concentration below $15 \mu \mathrm{g} / \mathrm{l}$ were not included in the comparative study, because reliable analysis of the fraction of prostate-specific antigen bound to concanavalin A-Sepharose is not possible (see results section).

\section{Methods}

Concanavalin A-Sepharose (100 ml, code 17-0440-01, Pharmacia, Woerden, NL) or Sepharose CL-4B (100 ml, code 17-0150-01, Pharmacia) was washed five times with $100 \mathrm{ml}$ buffer $(0.5 \mathrm{~mol} / \mathrm{l}$ $\mathrm{NaCl}, 1 \mathrm{mmol} / \mathrm{l} \mathrm{MnCl}, 1 \mathrm{mmol} / 1 \mathrm{MgCl}_{2}$ and $1 \mathrm{mmol} / \mathrm{l} \mathrm{CaCl}$ and $0.1 \mathrm{~mol} / \mathrm{l}$ sodium acetate buffer, $\mathrm{pH} 6.5$ ) and the remaining concanavalin A-Sepharose, after suction over a Whatman (GF/D) filter, was suspended in $75 \mathrm{ml}$ buffer. Varying amounts of concanavalin A-Sepharose or Sepharose $4 \mathrm{~B}$ were pipetted into tubes and the supernatant was removed after centrifugation for $10 \mathrm{~min}$ at 6000 $\mathrm{min}^{-1}$. The volume of the remaining swollen gel was determined ( $4 \mathrm{ml}$ contained $2.8 \mathrm{ml}$ completely swollen gel; the completely swollen gel contained about 6.8 gram dry concanavalin A-Sepharose per $100 \mathrm{mil}$ ). After adding serum or diluted serum to the completely swollen gel, the mixture was incubated at room temperature for 2 hours with gentle shaking on an overhead rotator and centrifuged as indicated before. The supernatant was aspirated for analysis of prostate-specific antigen and albumin.

Prostate-specific antigen was determined on a Stratus analyser (Baxter, Maarssen, NL) using a Fluorometric Enzyme İmmunoassay (Hybritech, Ely Lilly, Nieuwegein, NL). Dilutions were performed with the High Sample Diluent (buffer solution containing bovine serum albumin) in the testkit.

Albumin was determined on a Cobas Fara analyser (Roche, Mijdrecht, NL) using the bromocresol green method (Instruchemie, Hilversum, NL).

\section{Statistical analysis}

A paired Student's t-test was used for comparison of the results.

\section{Results and Discussion}

Optimization of the method

Correction for serum dilution by addition of swollen concanavalin A-Sepharose

Pre-diluted samples of $0.5 \mathrm{ml}$ from a patient with malignant prostatic disease (prostate-specific antigen 1235 $\mu \mathrm{g} / \mathrm{l}$, diluted 25 times with diluent before incubation), were incubated with varying amounts $(0.15 ; 0.3 ; 0.6$; $1.2 ; 2.4 \mathrm{ml}$ ) of completely swollen concanavalin $\mathrm{A}=\mathrm{Se}-$ pharose or Sepharose 4B. Barak et al. (3) used a separate experiment with Sepharose 4B to correct for the dilution introduced by adding the concanavalin A-Sepharose. The glycosylation variants of prostate-specific antigen do not bind to Sepharose 4B; the fraction of non-binding prostate-specific antigen was calculated as follows:

$\%$ non-bound prostate-specific antigen

$$
=100 \times \frac{\text { prostate-specific antigen in serum treated with concanavalin A-Sepharose }}{\text { prostate-specific antigen in serum treated with Sepharose 4B }}
$$

From figure 1 it can be concluded that albumin (nonglycosylated protein) does bind to concanavalin A-Sepharose or to Sepharose 4B. The dilution of serum caused by addition of concanavalin A-Sepharose was also determined by measuring the serum albumin concentration before and after addition of concanavalin A-
Sepharose. Experimental error is smaller when the dilution factor is obtained this way, rather than by performing a separate experiment with Sepharose 4B. Therefore, we used the dilution of serum albumin to correct for the dilution caused by addition of swollen gel.

$\%$ non-bound prostate-specific antigen

$$
=100 \times \frac{\text { prostate-specific antigen in serum treated with concanavalin A-Sepharose }}{\text { prostate-specific antigen in serum }} \times \text { dilution factor. }
$$




$$
\text { Dilution factor }=\frac{\text { albumin in serum }}{\text { albumin in serum treated with concanavalin A-Sepharose }}
$$

Prostate-specific antigen bound to concanavalin A-Sepharose $(\%)=100-$ prostate-specific antigen non-bound (\%).

\section{Effect of serum protein concentration on binding of prostate-specific antigen to concanavalin A-Sepharose}

A serum sample with a prostate-specific antigen concentration of $3250 \mu \mathrm{g} / \mathrm{l}$ was pre-diluted with a nil serum (serum containing no prostate-specific antigen) or with diluent (containing bovine serum albumin, a non-glycoprotein). Pre-dilution with the diluent diminishes the glycoprotein concentration of the sample. As can be seen in figure 2, optimal binding is obtained at a much lower concanavalin A concentration for samples pre-diluted with diluent than for samples pre-diluted with the nil serum. For measurements in serum (diluted with nil serum) at least $2.5 \mathrm{ml}$ completely swollen concanavalin A-Sepharose is required to allow optimal binding of prostate-specific antigen, e.g. to reach a situation where binding is independent of the serum glycoprotein concentration. As concanavalin A binding sites are also occupied by other glycoproteins in the serum sample, fewer concanavalin A binding places are required when using sera pre-diluted with the diluent. Figure 2 shows that for undiluted serum samples at least $2.5 \mathrm{ml}$ completely swollen gel is required to provide sufficient concanavalin A binding sites. When sub-optimal concanav-

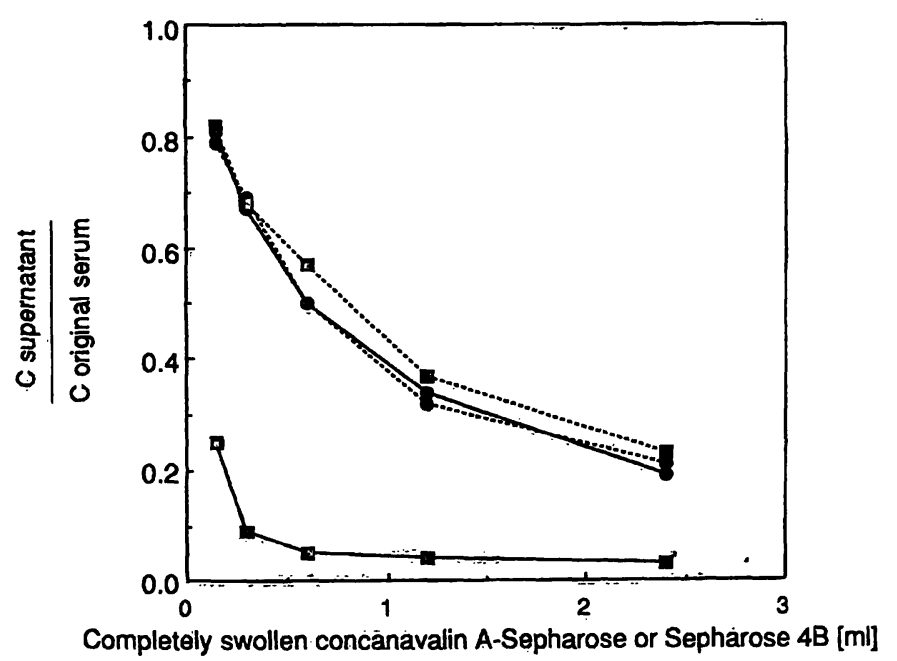

Fig. 1. Samples $(0.5 \mathrm{ml})$ from a patient with malignant prostatic disease (prostate-specific antigen: $1235 \mu \mathrm{g} / \mathrm{l}$, pre-diluted 25 times with diluent) were incubated with increasing amounts of completely swollen Sepharose 4B (๑) or concanavalin A-Sepharose (घ); the supernatant was removed after centrifugation.

The ratio, i. e. the concentration non-bound to Sepharose or to concanavalin A-Sepharose in the supernatant ( $\left.c_{\text {supematant }}\right)$ divided by the original sample eoncentration ( $c_{\text {original senum }}$ ) is depicted for both the non-glycosylated protein albumin (---) and for the glycosylated protein prostate-specific antigen (-).

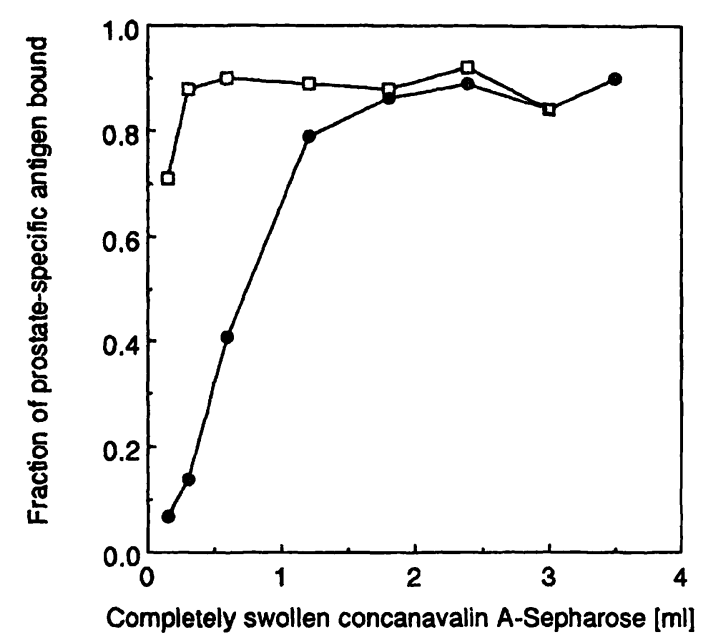

Fig. 2 Serum of a patient with malignant prostatic disease (prostate-specific antigen: $3250 \mu \mathrm{g} / \mathrm{l}$ ) was pre-diluted with a diluent $(\square)$ or with a nil serum containing no prostate-specific antigen $(0)$. Subsequently $0.5 \mathrm{ml}$ samples were added to different amounts of completely swollen concanavalin A-Sepharose.

Correction was made for dilution caused by addition of the swollen gel by measuring albumin before and after addition of concanavalin $\mathrm{A}$-Sepharose to the samples.

alin A binding sites are available, pre-diluted samples (samples with high prostate-specific antigen concentrations, mostly patients with carcinoma of the prostate) may give higher binding percentages than samples from benign prostatic hyperplasia patients, containing less prostate-specific antigen and needing no pre-dilution. This might erroneously suggest differences in the glycosylation pattern of prostate-specific antigen for patients with benign prostatic hyperplasia compared with patients with carcinoma of the prostate.

Figure 3 presents the binding curves for three serum specimens of prostate carcinoma patients with different prostate-specific antigen concentrations. For all three specimens the optimal amount of concanavalin A-Sepharose appeared to be at least $2.8 \mathrm{ml}$ of completely swollen gel. Neither Barak et al. (3) nor Chan \& Gao (4), who advocated the use of concanavalin A binding to prostate-specific antigen to discriminate between patients with benign prostatic hyperplasia or prostatic carcinoma, described control experiments to investigate whether sufficient concanavalin A binding sites were present during their experiments. From our experiments it can be concluded that with $0.5 \mathrm{ml}$ undiluted serum, at least $2.5 \mathrm{ml}$ completely swollen concanavalin A-Sepharose should be used to obtain maximal binding of 


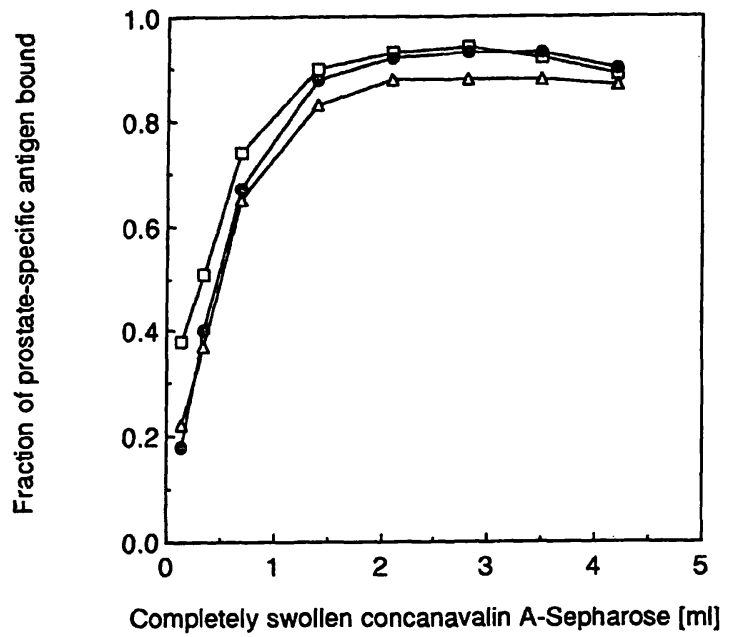

Fig. 3 Sera $(0.5 \mathrm{ml})$ of three patients with prostate-specific antigen concentrations of $770 \mu \mathrm{g} / \mathrm{l}(\square), 385 \mu \mathrm{g} / \mathrm{l}(0)$ and $198 \mu \mathrm{g} / \mathrm{l}(\Delta)$ prostate-specific antigen were incubated with increasing amounts of completely swollen concanavalin A-Sepharose.

To ensure maximal binding at least $2.5 \mathrm{ml}$ completely swollen concanavalin A-Sepharose is required for $0.5 \mathrm{ml}$ serum (undiluted).

the glycosylation variants of prostate-specific antigen. Moreover, a correction should be made for the dilution of serum due to the addition of gel (see fig. 1). Both Barak et al. (3) and Chan \& Gao (4) used a $1: 1$ ratio of serum to concanavalin A-Sepharose, which probably leads to the addition of insufficient binding sites (fig. 3).

The optimized procedure for measuring patient samples therefore is:

Serum ( $0.5 \mathrm{ml}$, not pre-diluted) is added to $2.8 \mathrm{ml}$ completely swollen concanavalin A-Sepharose. After adding serum to the gel the mixture is incubated at room temperature for 2 hours with gentle shaking on an overhead rotator and subsequently centrifuged. The supernatant is aspirated for analysis of prostate-specific antigen and albumin, from which a correction for dilution can be made; see the calculation of the percentage prostatespecific antigen bound to concanavalin A-Sepharose (above).

To allow comparison with other studies, we also analysed the samples with the sub-optimal procedure. Here, $0.5 \mathrm{ml}$ serum (not pre-diluted) is added to $0.5 \mathrm{ml}$ completely swollen concanavalin A-Sepharose. The final part of the procedure is similar to the optimized procedure described above.

\section{Precision}

The detection limit of the prostate-specific antigen determination is $0.24 \mu \mathrm{g} / \mathrm{l}$, which implies that for the optimized procedure $(2.8 \mathrm{ml}$ swollen gel and $0.5 \mathrm{ml}$ serum $)$ at $0 \%, 90 \%$ and $95 \%$ binding, the theoretical minimal serum concentration for detection of prostate-specific antigen in the supernatant after incubation with the gel should be respectively $1.3,13.0$ and $25.9 \mu \mathrm{g} / 1$ prostatespecific antigen. This indicates that in this procedure, for binding percentages of $90 \%$, sera should theoretical contain at least $13 \mu \mathrm{g} / \mathrm{l}$ prostate-specific antigen.

$$
\text { : } 1
$$

Precision was determined by measuring the percentage of concanavalin A-Sepharose binding for three sera with different prostate-specific antigen concentrations. For each serum the percentage prostate-specific antigen bound was determined from ten separate binding experiments. For each experiment $0.5 \mathrm{ml}$ serum was added to $2.8 \mathrm{ml}$ of completely swollen gel. Mean serum prostatespecific antigen concentrations were 12.2, 33.8 and 83.7 $\mu \mathrm{g} / \mathrm{l}$ and the percentages bound to concanavalin A-Sepharose were respectively $73.7 \pm 16.3 \% ; 86.9 \pm 3.3 \%$ and $87.4 \pm 2.3 \%$ (mean $\pm \mathrm{CV}$ ). A large experimental error is observed for the samples with serum prostatespecific antigen concentrations lower than $15 \mu \mathrm{g} / 1$, especially when high percentage binding of prostatespecific antigen to concanavalin A-Sepharose is expected. Assay variations were not given in earlier studies on this subject.

For a sample containing $15 \mu \mathrm{g} / \mathrm{l}$, addition of concanavalin A results in a decrease of prostate-specific antigen to less than $3 \mu \mathrm{g} / \mathrm{l}$ prostate-specific antigen. When $80-$ $90 \%$ of the prostate-specific antigen binds to the concanavalin $\mathrm{A}$, as can be expected, concentrations below $0.3 \mu \mathrm{g} / \mathrm{l}$ prostate-specific antigen have to be detected. Therefore these experiments cannot be performed on samples with prostate-specific antigen concentrations below $15 \mu \mathrm{g} / \mathrm{l}$, because the assay variation becomes too large. This makes the method unsuitable for discrimination of benign prostatic hyperplasia and carcinoma patients with prostate-specific antigen concentrations below $15 \mu \mathrm{g} / 1$.

Tab. 1 Comparison of the optimal $(2.8 \mathrm{ml}$ swollen concanavalin A-Sepharose) and the sub-optimal ( $0.5 \mathrm{ml}$ swollen concanavalin A-Sepharose) binding assay used to separate the glycosylation variants of prostate-specific antigen in patients with benign prostatic hyperplasia or carcinoma of the prostate.

\begin{tabular}{lllll}
\hline & \multicolumn{3}{l}{$\begin{array}{l}\text { Fraction of prostate- } \\
\text { specific antigen bound to } \\
\text { concanavalin A-Sepharose (\%) }\end{array}$} \\
\cline { 2 - 5 } & mean & SD & $\min$ & $\max$ \\
\hline $\begin{array}{l}\text { Benign prostatic } \\
\text { hyperplasia group, } n=7\end{array}$ & & & & \\
$\quad$ Sub-optimal assay & 50.4 & 8.3 & 37.0 & 63.0 \\
$\quad$ Optimal assay & 86.3 & 7.5 & 73.0 & 95.0 \\
$\quad$ & & & & \\
Prostatic carcinoma & & & & \\
group, $n=14$ & & & & \\
$\quad$ Sub-optimal assay & 51.6 & 9.5 & 37.0 & 65.0 \\
Optimal assay & 81.8 & 12.0 & 62.0 & 97.0 \\
\hline
\end{tabular}




\section{Clinical evaluation}

Patients with prostate-specific antigen concentrations below $15 \mu \mathrm{g} / \mathrm{l}$ were eleminated from the study, because reliable analysis of the percentage prostate-specific antigen bound to concanavalin A-Sepharose is not possible. Therefore only 7 benign prostatic hyperplasia (52.8 $\pm 50.1 \mu \mathrm{g} / 1$ prostate-specific antigen, mean $\pm \mathrm{SD}$ ) and 14 prostatic carcinoma patients $(1280 \pm 4130 \mu \mathrm{g} / \mathrm{l}$ prostate-specific antigen, mean \pm SD) were included in the comparative study. The prostate-specific antigen concentrations of the two carcinoma patients eliminated were respectively 8.9 and $13.9 \mu \mathrm{g} / \mathrm{l}$. The mean prostatespecific antigen concentration of the 72 benign prostatic hyperplasia patients with prostate-specific antigen concentrations lower than $15 \mu \mathrm{g} / \mathrm{l}$, that were not included in the comparative study, was $2.9 \pm 2.5 \mu \mathrm{g} / 1$ (mean $\pm \mathrm{SD}$ ).

Figure 4 presents the distribution of the percentages of concanavalin A-Sepharose-bound prostate-specific antigen in prostatic cancer and in benign prostatic hyperplasia patients. Sera $(0.5 \mathrm{ml})$ from prostate cancer patients and from benign prostatic hyperplasia patients were treated with $2.8 \mathrm{ml}$ completely swollen concanava' lin A-Sepharose. To allow comparison with earlier studies, all samples were also measured under sub-optimal binding conditions, where $0.5 \mathrm{ml}$ serum was added to $0.5 \mathrm{ml}$ completely swollen concanavalin A-Sepharose. The percentage prostate-specific antigen bound to concanavalin A-Sepharose was determined as described earlier.

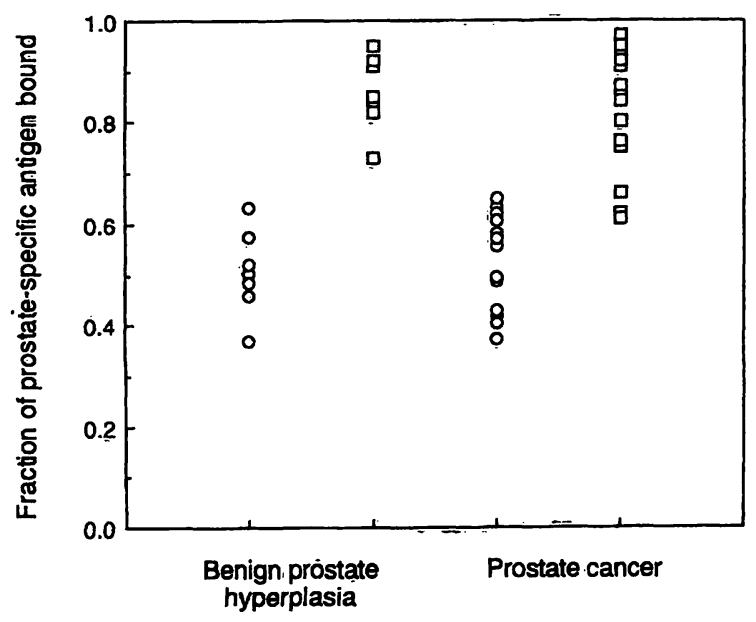

Fig. 4 Distribution of the percentage of prostate-specific antigen bound to concanavalin A-Sepharose in patients with prostate cancer (14) or benign prostate hyperplasia (7) having prostate-specific antigen concentrations higher than $15 \mu \mathrm{g} /$.

Both the optimal binding assay ( $2.8 \mathrm{ml}$ concanavalin A-Sepharose (D)) and the sub=optimal binding assay $(0.5 \mathrm{ml}$ concanavalin ASepharose (O)) were used.
With the optimized procedure $(2.8 \mathrm{ml}$ concanavalin ASepharose) no significant differences in prostate-specific antigen glycosylation patterns were observed between benign prostatic hyperplasia and prostatic carcinoma patients. The sub-optimal procedure also showed no significant differences between benign prostatic hyperplasia and carcinoma patients. since different pre-treatments can lead to results that glycosylation between benign prostatic hyperplasia and prostate carcinoma patients, all samples underwent the same pre-treatment, i. e. no difference in the pre-dilution of samples from carcinoma patients and benign prostatic hyperplasia patients.

Highly significant differences $(p<0.0001)$ were found with the optimal binding assay $(2.8 \mathrm{ml}$ concanavalin ASepharose) but not with the sub-optimal binding assay ( $0.5 \mathrm{ml}$ concanavalin A-Sepharose).

From the data presented we suggest that the discrepancies in the literature are probably caused by the use of insufficient concanavalin A binding capacity $(3,4)$, the lack of correction (4), or incorrect evaluation of the dilution effect caused by addition of concanavalin A-Sepharose (5) or pre-dilution of samples with high prostate-specific antigen concentrations (carcinoma group), in contrast to the samples of patients with benign prostatic hyperplasia. Moreover, none of the studies report the lower detection limit or the analytical variation, especially for prostate-specific antigen concentrations below $15 \mu \mathrm{g} / \mathrm{l}$, where an extremely large experimental error is to be expected when the percentage of prostatespecific antigen bound to concanavalin A-Sepharose is higher than $90 \%$.

From the results we conclude that when using optimal binding conditions (i. e. maximal binding of $\mathrm{N}$-glycosylation variants of prostate-specific antigen to concanavalin A-Sepharose and no pre-dilution of samples with high prostate-specific antigen concentrations), no significant differences in binding ratio can be demonstrated between patients with benign prostatic hyperplasia and patients with prostatic carcinoma.

In general, there is a danger of drawing invalid conclusions from measurement of the lectin binding of glycoproteins in serum, since non-covalent lectin-carbohydrate interactions are influenced by a number of different experimental variables.

\section{Acknowledgements}

We thank Mia van der Vloet-Boermans for her perfect technical assistance. 


\section{References}

1. Gittes, R. F. (1991) Carcinoma of the prostate (review). New Engl. J. Med. 24, 236-245.

2. Killian, C. S. \& Ming Chu, T. (1990) Prostate-specific antigen: Questions often asked. Cancer Investigation 8, 27-37.

3. Barak, M., Mecz, Y., Lurie, A. \& Gruener, N. (1989) Binding of serum prostate antigen to concanavalin $A$ in patients with cancer or hyperplasia of the prostate. Oncology 46, 375-377.

4. Chan, D. W. \& Gao, Y. M. (1991) Variants of prostate-specific antigen separated by concanavalin A. Clin. Chem. 37, 11331134.

5. Marrink, J., Klip, H. \& De Jong, R. (1992) Prostate-specific antigen-con $\mathrm{A}$ binding ratio in benign prostate hyperplasia and prostate cancer. The Lancet 339, 619-620.
6. Van Pelt, J. \& Van Dieijen-Visser, M. P. (1992) PSA-con-A binding ratio in benign prostate hyperplasia and prostate cancer. Letter. The Lancet 340, 174-175.

7. Tumer, G. A. (1992) N-Glycosylation of serum proteins in disease and its investigation using lectins. Clin. Chim. Acta 208, $149-171$.

Prof. dr. M. P. van Dieijen-Visser Department of Cliniçal Chemistry Academic Hospital Maastricht P. O. Box.

NL-6202 AZ Maastricht

The Netberlands 